\title{
Catheter-Induced 3:1 Second Degree Atrioventricular Nodal Block During Atrioventricular Nodal Reentrant Tachycardia
}

\author{
Leandro Zimerman, Claudio Medeiros, Gustavo Lima
}

Porto Alegre, RS - Brazil

Second-degree atrioventricular (AV) nodal block with 2:1 AV conduction during atrioventricular nodal reentrant tachycardia (AVNRT) has been previously described. We report a patient who developed AVNRT during which 3:1 intra-infra-Hisian block due to catheter manipulation was observed. This was followed by gradual, incremental recovery of AV conduction.

AVNRT accounts for approximately $50 \%$ of cases of supraventricular tachycardia. This mechanism of tachycardia uses a reentrant circuit in the region of the AV Node which usually contains distinct slow and fast pathways. Radiofrequency ablation of the slow pathway is an effective and safe treatment for AVNRT ${ }^{1}$.

In this report, we present a patient with AVNRT of the common type who developed 3:1 intra-infra-Hisian block during AVNRT as a result of catheter manipulation. Before resumption of normal 1:1 conduction, we observed split His potentials in normal sinus rhythm, followed by atrioventricular (AV) nodal echo beats with intra-infra-Hisian block.

\section{Case Report}

An 81-year-old white female was referred for evaluation of medically refractory supraventricular tachycardia. At electrophysiologic study, catheters were advanced to the right ventricular apex, His bundle area, high right atrium and the coronary sinus without difficulty. After obtaining basic intervals (PA:47msec; $\mathrm{AH}$ : 40msec; HV: $53 \mathrm{msec}$ ), right atrial and right ventricular premature stimulation and decremental pacing were performed without induction of tachycardia. With a basic drive train of $600 \mathrm{msec}$, the AV nodal efective refractory period was $<340 \mathrm{~ms}$.

Administration of isoproterenol $3 \mu \mathrm{g} / \mathrm{min}$ and atropine $0.6 \mathrm{mg}$ intravenously caused the heart rate to increase from $57 \mathrm{bpm}$ to $75 \mathrm{bpm}$. Programmed atrial extrastimulation with basic drive of $600 \mathrm{msec}$ showed that the effective refractory period of the fast and slow AV nodal pathways were $380 \mathrm{msec}$ and $<330 \mathrm{msec}$ respectively. Right atrial burst pacing subsequently induced sustained AVNRT of the

From the Division of Cardiology, Hospital de Clínicas de Porto Alegre, RS Mailing adress: Leandro Zimerman - Av. Iguassu, 176/402 - 90470-430 - Porto Alegre, RS - Brazil common type with 3:1 intra-infra-Hisian block (fig. 1). The atrial cycle length during tachycardia was $280 \mathrm{msec}$, while the ventricular cycle length was $840 \mathrm{msec}$. Evidence supporting AVNRT as the mechanism of tachycardia are as follows: 1) dual AV nodal physiology was present; 2) retrograde atrial activation was concentric during ventricular pacing and during tachycardia; 3 ) premature ventricular beats introduced when the His bundle was refractory did not preexcite the atrium; 4) the tachycardia was induced with a critical AV delay; 5) the surface V to His A interval during reciprocating tachycardia was $-15 \mathrm{msec} ; 6$ ) tachycardia persisted during 3:1 conduction, showing that the ventricle was not a critical component of the circuit; 7) application of radiofrequency current to the posteroseptal right atrium resulted in noninducibility of the tachycardia. Isoproterenol was discontinued, and the tachycardia was terminated with right ventricular burst pacing.

During normal sinus rhythm, 1:1 AV nodal conduction returned. However, the His bundle electrogram was notable for a split His depolarization (Figure 2). The basic intervals were as follows: PA, 45msec; AH, 47msec; HH', 34msec; $\mathrm{H}^{\prime} \mathrm{V}, 38 \mathrm{msec}$. The split His potentials resolved after one hour, at which point right atrial burst pacing induced AV nodal echo beats with intra-infra-Hisian block(Figure 3). Subsequently, right atrial extrastimuli and decremental pacing were repeated, and no abnormalities in His bundle conduction were noted. AVNRT with 1:1 HV conduction was then induced with right atrial pacing. Six applications of radiofrequency current were then delivered to the posteroseptal right atrium, and junctional rhythm was noted during the final application. AVNRT was no longer inducible, and $\mathrm{AV}$ nodal conduction time was normal during sinus rhythm (PA: 49msec; AH: 49msec; HV: 50msec).

\section{Discussion}

In previous reports, $2: 1$ infranodal conduction block during AVNRT has been rarely observed, usually appearing immediately after induction of AVNRT and lasting for a few beats ${ }^{1-5}$. All but one ${ }^{5}$ of the cases of second degree AV block ${ }^{1-5}$ and atrioventricular dissociation ${ }^{3}$ during AVNRT occurred during AVNRT of the common type. Sites of block in previous reports have been above the AV node ${ }^{1,3}$, above the His bundle ${ }^{1,4,5}$, and intra-infra-His bundle ${ }^{1,2}$. Block 


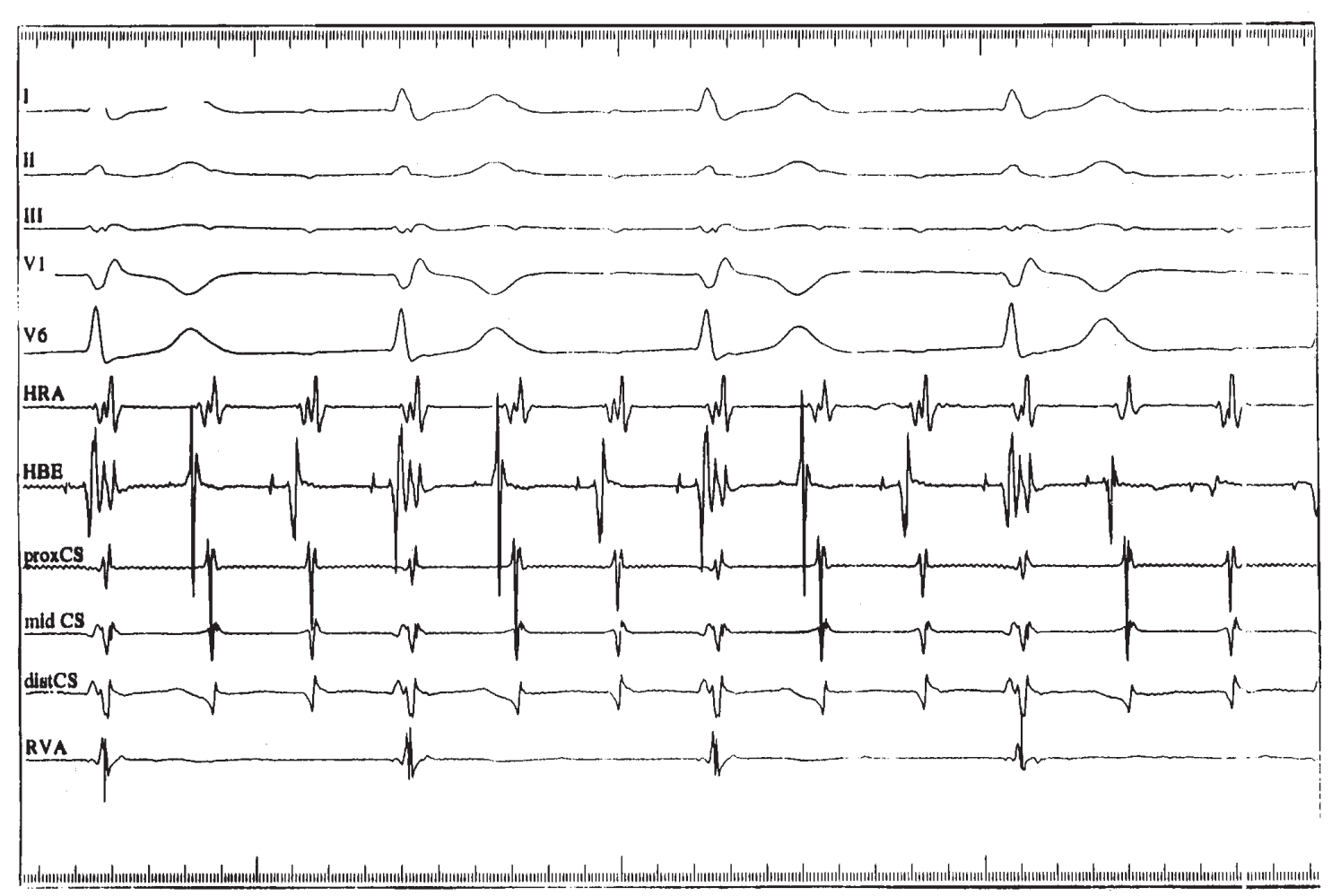

Fig. 1 - AVNRT of the common type with 3:1 conduction block to the ventricles and 1:1 concentric retrograde atrial activation. Despite the presence of AVN block, the tachycardia is sustained. I, II, III, V1 and V6- surface leads; HRA- high right atrium; HBE- His bundle; prox CS- proximal coronary sinus; mid CS- middle coronary sinus; dist CS- distal coronary sinus; RVA- right ventricular apex.

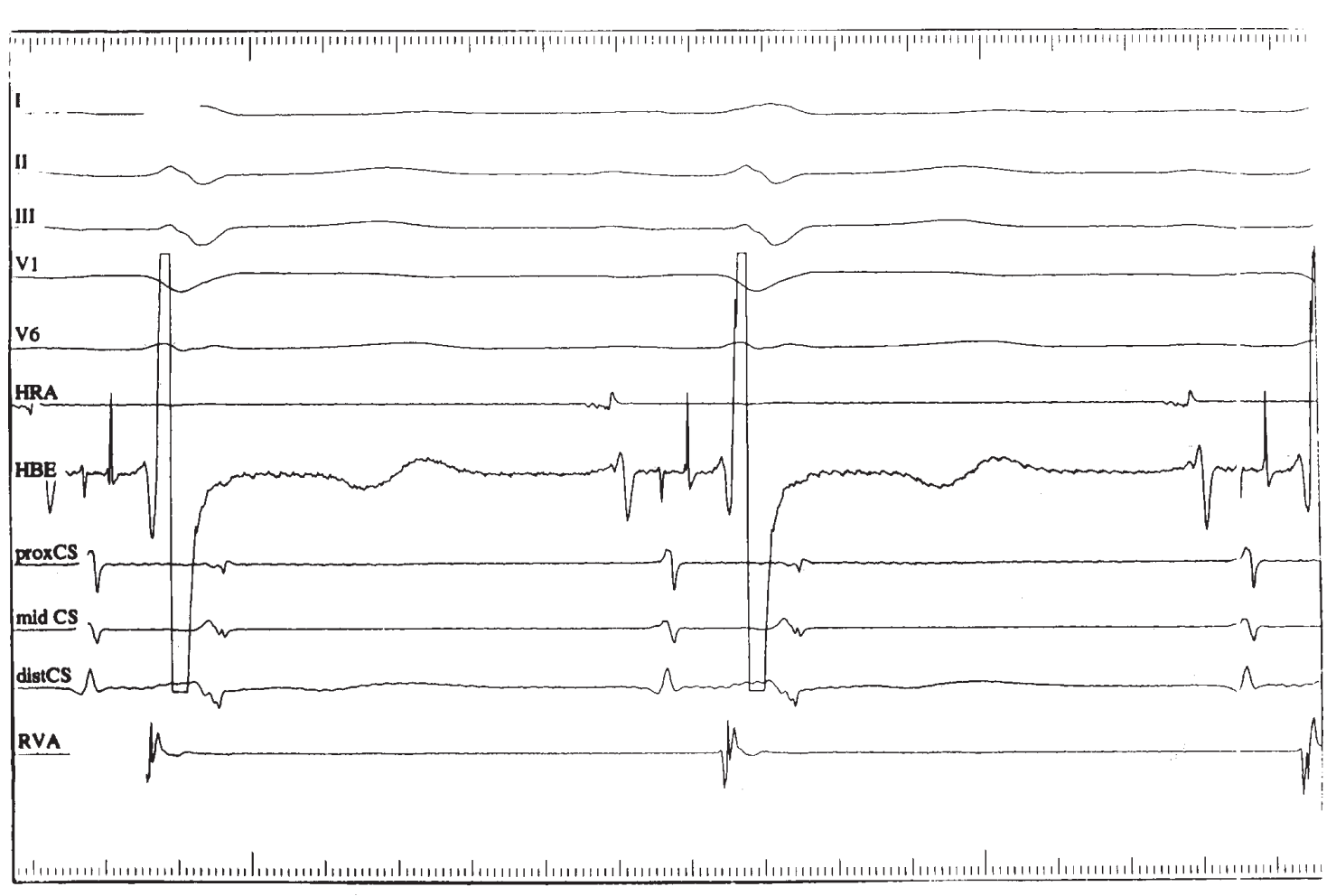

Fig. 2 - Split His is observed during normal sinus rhythm after tachycardia termination. The measurements in the His catheter are as follows: AH:47ms; HH':34ms; HV:38ms. I, II, III, V1 and V6- surface leads; HRA- high right atrium; HBE- His bundle; prox CS- proximal coronary sinus; mid CS- middle coronary sinus; dist CS- distal coronary sinus; RVAright ventricular apex. 


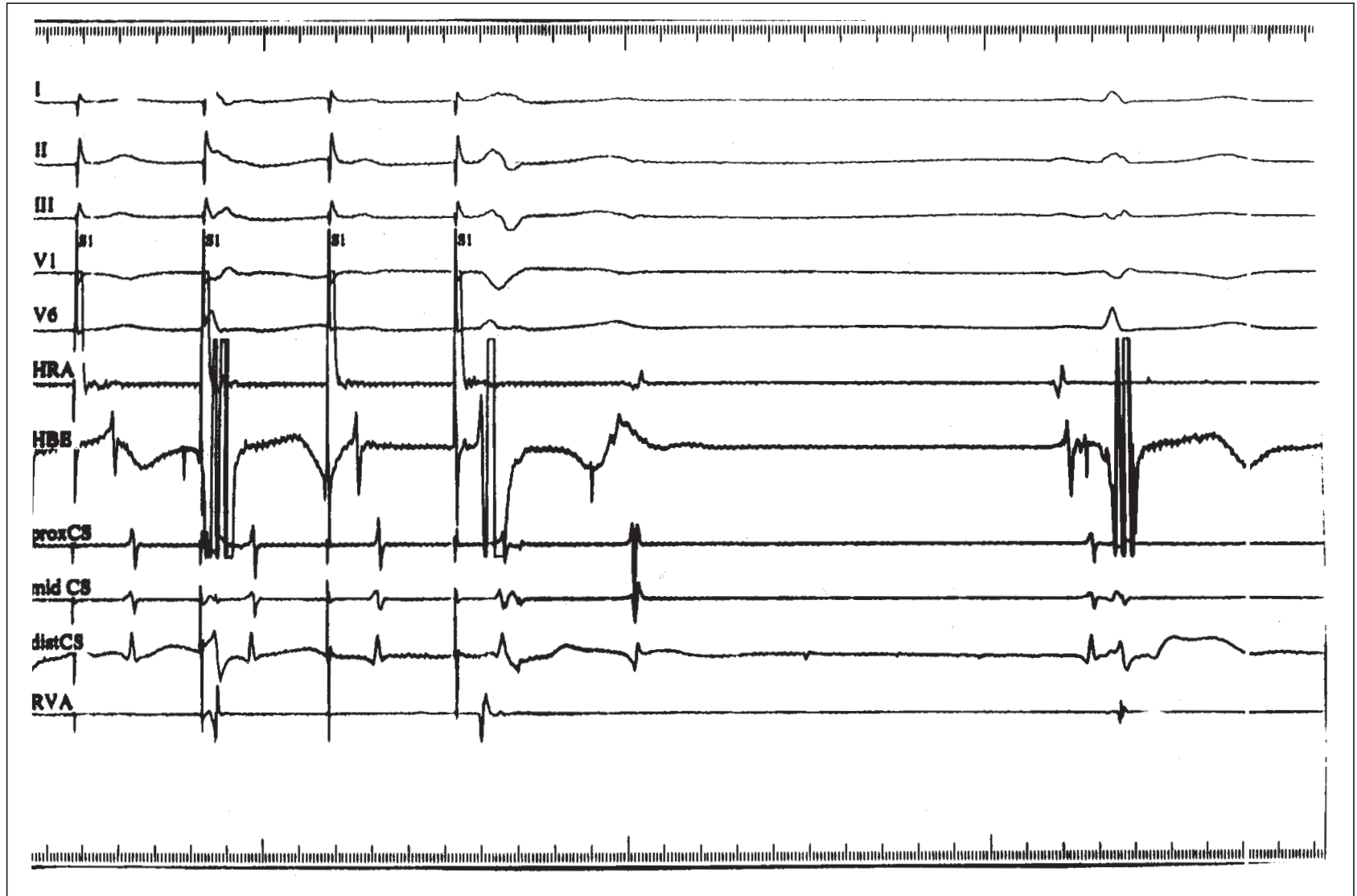

Fig. 3 - Atrioventricular nodal reentry echo beat with the same retrograde atrial activation seen in figure 1 (during tachycardia) and with infra-Hisian block observed at the end of atrial burst pacing with a cycle length of $350 \mathrm{~ms}$. This confirms that the atrioventricular conduction is still abnormal, despite the absence of split His in the sinus beat. S1: atrial stimuli. I, II, III, V1 and V6: surface leads; HRA- high right atrium; HBE- His bundle; prox CS- proximal coronary sinus; mid CS- middle coronary sinus; dist CS- distal coronary sinus; RVA- right ventricular apex.

persisting for almost one hour with subsequent incremental recovery has not been previously reported. We are also unaware of any previous reports of high-degree $3: 1 \mathrm{AV}$ conduction block during AVNRT.

This patient developed a 3:1 intra-infra-Hisian block during AVNRT of the common type that was present for one hour. The most probable cause of the block in this case was catheter manipulation. Conduction block during AVNRT attributed to His bundle catheter manipulation has been previously described ${ }^{6}$, but the site of block was above the His bundle. During AVNRT with 3:1 block, we observed an $\mathrm{H}$ potential on the His bundle recordings, with a fixed HA interval. This is suggestive of an infra-Hisian point of block. However, since a split His depolarization was noted after restoration of normal conduction, intra-Hisian, rather than infra-Hisian, block is more likely. Interestingly, the tachycardia with 3:1 block was terminated with right ventricular burst pacing, suggesting that the conduction block was antegrade only.

In summary, this case is notable for the novel observation of prolonged 3:1 infranodal conduction block during AVNRT. The site of block was either intra or infra-Hisian and possibly only antegrade. Block was followed by incremental recovery of conduction.

\section{References}

1. Haissaguerre M, Gaita F, Fischer B, et al - Elimination of atrioventricular reentrant tachycardia using discrete slow potentials to guide application of radiofrequency energy. Circulation 1992; 85: 2162-75

2. Wellens HJ, Wesdorp JC, Duren DR, Lie KI - Second degree block during reciprocal atrioventricular nodal tachycardia. Circulation 1976; 53: 595-9.

3. Vassalo JA, Cassidy DM, Josephson ME - Atrioventricular nodal supraventricular tachycardia. Am J Cardiol 1985; 56: 193-5.

4. Portillo B, Mejias J, Leon-Portillo N, et al - Entrainment of atrioventricular nodal reentrant tachycardias during overdrive pacing from high right atrium and coronary sinus - with special reference to atrioventricular dissociation and 2:1 retrograde block during tachycardias. Am J Cardiol 1984; 53: 1570-5.

5. DiMarco JP, Sellers TD, Belardinelli L - Paroxysmal supraventricular tachycardia with Wenckebach block: evidence for reentry within the upper portion of the atrioventricular node. J Am Coll Cardiol 1984; 3: 1551-5.

6. Ko PT, Nacarelli GV, Gulamhusein S, et al - Atrioventricular dissociation during paroxysmal junctional tachycardia. PACE 1981; 4: 670-7. 